\title{
Measurement of the branching ratios $\psi^{\prime} \rightarrow e^{+} e^{-}, \psi^{\prime} \rightarrow J / \psi \pi \pi$ and $\psi^{\prime} \rightarrow J / \psi \eta$.
}

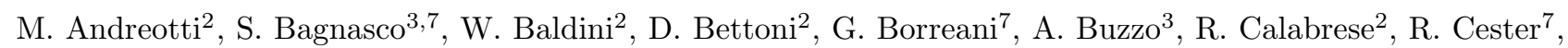
G. Cibinetto ${ }^{2}$, P. Dalpiaz² ${ }^{2}$ G. Garzoglio ${ }^{1}$, K. E. Gollwitzer ${ }^{1}$, M. Graham ${ }^{5}$, M. Hu ${ }^{1}$, D. Joffe ${ }^{6}$, J. Kasper ${ }^{6}$, G. Lasio $^{4}$, M. Lo Vetere ${ }^{3}$, E. Luppi ${ }^{2}$, M. Macrì ${ }^{3}$, M. Mandelkern ${ }^{4}$, F. Marchetto ${ }^{7}$, M. Marinelli ${ }^{3}$, E. Menichetti ${ }^{7}$, Z. Metreveli ${ }^{6}$, R. Mussa ${ }^{7}$, M. Negrini ${ }^{2}$, M. M. Obertino ${ }^{7}$, M. Pallavicini ${ }^{3}$, N. Pastrone ${ }^{7}$, C. Patrignani ${ }^{3}$, T. K. Pedlar ${ }^{6}$, S. Pordes ${ }^{1}$, E. Robutti ${ }^{3}$, W. Roethel ${ }^{6,4}$, J. L. Rosen ${ }^{6}$, P. Rumerio ${ }^{6}$, R. Rusack ${ }^{5}$, A. Santroni ${ }^{3}$, J. Schultz ${ }^{4}$, S. H. Seo ${ }^{5}$, K. K. Seth ${ }^{6}$, G. Stancari ${ }^{1}$, M. Stancari ${ }^{4}$, A. Tomaradze ${ }^{6}$, I. Uman ${ }^{6}$, T. Vidnovic III ${ }^{5}$, S. Werkema ${ }^{1}$ and P. Zweber ${ }^{6}$

(FNAL experiment E835)

\author{
${ }^{1}$ Fermi National Accelerator Laboratory, Batavia, Illinois 60510 \\ ${ }^{2}$ Istituto Nazionale di Fisica Nucleare and University of Ferrara, 44100 Ferrara, Italy \\ ${ }^{3}$ Istituto Nazionale di Fisica Nucleare and University of Genova, 16146 Genova, Italy \\ ${ }^{4}$ University of California at Irvine, Irvine, California 92697 \\ ${ }^{5}$ University of Minnesota, Minneapolis, Minnesota 55455 \\ ${ }^{6}$ Northwestern University, Evanston, Illinois 60208 \\ ${ }^{7}$ Istituto Nazionale di Fisica Nucleare and University of Torino, 10125 Torino, Italy
}

(Dated: September 25, 2018)

\begin{abstract}
We have measured several branching ratios for $\psi^{\prime}$ decay using the data collected by FNAL E835 experiment during year 2000, obtaining $\mathcal{B}\left(\psi^{\prime} \rightarrow e^{+} e^{-}\right)=0.0068 \pm 0.0001 \pm 0.0004$, $\mathcal{B}\left(\psi^{\prime} \rightarrow J / \psi \pi^{+} \pi^{-}\right)=0.292 \pm 0.005 \pm 0.018, \mathcal{B}\left(\psi^{\prime} \rightarrow J / \psi \pi^{0} \pi^{0}\right)=0.167 \pm 0.005 \pm 0.014$ and $\mathcal{B}\left(\psi^{\prime} \rightarrow J / \psi \eta\right)=0.028 \pm 0.002 \pm 0.002$. We also present a measurement of the dipion mass distribution in the decays $\psi^{\prime} \rightarrow J / \psi \pi \pi$.

PACS numbers: 13.75.Cs, 14.40.Gx
\end{abstract}

\section{INTRODUCTION}

Branching ratios of $\psi^{\prime}$ decay have been measured by many experiments in $e^{+} e^{-}$collisions. The branching ratios to $e^{+} e^{-}$and to $J / \psi$ inclusive states have been measured also by the Fermilab experiments E760 [1] and E835 [2], which studied charmonium spectroscopy in $\bar{p} p$ annihilation.

The strong $\psi^{\prime}$ decays to $J / \psi$ proceed mainly through the emission of soft gluons by the $\bar{c} c$ pair, with their subsequent hadronization [3]. The low gluon momentum makes this process nonperturbative. However some features of the hadronic process $\psi^{\prime} \rightarrow J / \psi X$ can be predicted using conservation laws. A prediction based on isospin conservation is that $\mathcal{B}\left(\psi^{\prime} \rightarrow J / \psi \pi^{0} \pi^{0}\right) / \mathcal{B}\left(\psi^{\prime} \rightarrow\right.$ $\left.J / \psi \pi^{+} \pi^{-}\right)=\frac{1}{2}$. While previous measurements have always yielded slightly larger values, further checks of this ratio are needed. The multipole expansion of the gluon field 4] has been used to predict a value in the range 0.10 - 0.14 for $\Gamma\left(\psi^{\prime} \rightarrow J / \psi \eta\right) / \Gamma\left(\psi^{\prime} \rightarrow J / \psi \pi^{+} \pi^{-}\right)$5, 6] and the $\pi \pi$ invariant mass distribution 7,8 ].

\section{EXPERIMENTAL METHOD}

E835 studies charmonium spectroscopy in $\bar{p} p$ annihilation. All $\bar{c} c$ states can be directly formed with this technique. Since the cross section for charmonium formation is at best $\sim 5$ orders of magnitude smaller than the total $\bar{p} p$ cross section, in order to improve the signal to noise ratio the charmonium signal is extracted from the hadronic background by detecting electromagnetic final states.

$\psi^{\prime}$ decays are studied here by selecting events with a high invariant mass $e^{+} e^{-}$pair in the final state, coming from the reactions:

$$
\begin{aligned}
& \psi^{\prime} \rightarrow e^{+} e^{-}, \\
& \psi^{\prime} \rightarrow J / \psi \pi^{+} \pi^{-} \rightarrow e^{+} e^{-} \pi^{+} \pi^{-}, \\
& \psi^{\prime} \rightarrow J / \psi \pi^{0} \pi^{0} \rightarrow e^{+} e^{-} 4 \gamma, \\
& \psi^{\prime} \rightarrow J / \psi \eta \rightarrow e^{+} e^{-} 2 \gamma
\end{aligned}
$$

The number $N_{A}$ of events observed for $\psi^{\prime}$ decay to a given final state $A$ is given by:

$N_{A}=\mathcal{L} \cdot\left(\sigma_{b k g}+\epsilon_{A} \cdot \int G(E) \cdot \sigma_{B W}(E) \cdot \mathcal{B}\left(\psi^{\prime} \rightarrow A\right) d E\right)$

where $\mathcal{L}$ is the integrated luminosity, $\sigma_{b k g}$ the background cross section, $\epsilon_{A}$ the overall detection efficiency for the channel, $\sigma_{B W}$ the Breit-Wigner cross section for $\bar{p} p \rightarrow \psi^{\prime}$, $G(E)$ the center of mass energy distribution and $\mathcal{B}\left(\psi^{\prime} \rightarrow\right.$ $A)$ the branching ratio for the decay $\psi^{\prime} \rightarrow A$.

Measuring the ratio of branching ratios for two channels $A$ and $B$ on the same data sample, several factors in (1) cancel, leading to:

$$
\frac{\mathcal{B}\left(\psi^{\prime} \rightarrow A\right)}{\mathcal{B}\left(\psi^{\prime} \rightarrow B\right)}=\frac{\epsilon_{B}}{\epsilon_{A}} \cdot \frac{N_{A}-N_{b k g, A}}{N_{B}-N_{b k g, B}},
$$

where $N-N_{b k g}$ is the number of events after the subtraction of the background contribution. The reference channel has been chosen to be the $J / \psi$ inclusive decay because it is the one for which the ratio (2) presents the lowest systematics, as will be shown in Section VI. 


\section{EXPERIMENTAL APPARATUS}

E835 9] is a fixed target experiment in which the $\bar{p}$ beam circulating in the Fermilab Antiproton Accumulator (AA) crosses an internal hydrogen gas jet target. During a typical data taking period the $\bar{p}$ are accumulated until a current of $\sim 70 \mathrm{~mA}$ is reached. Then accumulation stops and the $\bar{p}$ are stochastically cooled and decelerated to the energy of the resonance to be studied. At this point the $\mathrm{H}_{2}$ target is turned on and the data taking proceeds on the resultant "stack" until the $\bar{p}$ beam current is around $10 \mathrm{~mA}$. In this current range it is possible to take data at constant luminosity by regulating the jet target density.

After deceleration, the mean center of mass energy $E_{c m}$ of the $\bar{p} p$ system is known with a precision better than $\sim 100 \mathrm{keV}$, where the $E_{c m}$ distribution is gaussian with a $\sigma$ typically ranging from 200 to $400 \mathrm{keV}$. Further details about the AA operation during E835 data taking can be found in [10].

The E835 detector is a nonmagnetic spectrometer with cylindrical symmetry around the beam axis. The inner part of the detector is the charged tracking system; for the year 2000 data taking it was composed of three cylindrical hodoscopes, two straw chambers for the measurement of the azimuthal angle $\phi$ (around the beam axis), two scintillating fiber detectors for the measurement of the polar angle $\theta$ (with respect to the beam axis) and an additional hodoscope in the forward direction used as a charged veto. The three cylindrical hodoscopes are segmented in $\phi$ in 8 (for the inner), 24 (for the intermediate) and 32 (for the outer) modules respectively. The hodoscopes provide $d E / d x$ information for the charged tracks and they are used to form the charged hardware trigger.

A 16 cell Čerenkov counter with 8 azimuthal and two polar angle sections cover the full azimuth and the polar angle region $15^{\circ} \leq \theta \leq 65^{\circ}$ and allows the separation of high energy $e^{ \pm}$, mostly produced in $J / \psi$ and $\psi^{\prime}$ decay, from the other charged particles, which is used in the first level trigger.

Two electromagnetic calorimeters, the Central Calorimeter (CCAL) and the Forward Calorimeter (FCAL) cover the region $11^{\circ} \leq \theta \leq 70^{\circ}$ and $3^{\circ} \leq \theta \leq 11^{\circ}$ respectively. The CCAL energy resolution is $\bar{\sigma}_{E} / \bar{E}=1.4 \%+6 \% / \sqrt{E(G e V)}$ and its angular resolutions are $\sigma_{\phi} \sim 11 \mathrm{mrad}$ and $\sigma_{\theta} \sim 6 \mathrm{mrad}$. FCAL is not used in the following analysis.

The luminosity is measured with $\sim 2 \%$ accuracy by means of solid state detectors located below the interaction region at $\theta \simeq 90^{\circ}$ to the $\bar{p}$ beam.

\section{EVENT SELECTION}

In year 2000, E835 collected a total integrated luminosity of $\mathcal{L}=113 \mathrm{pb}^{-1}$ of data. The data sample used in this analysis consists of $14.4 \mathrm{pb}^{-1}$ collected in the $\psi^{\prime}$ resonance region, with $12.5 \mathrm{pb}^{-1}$ on the resonance and $1.9 \mathrm{pb}^{-1}$ in two samples at energies above $(3704.9 \mathrm{MeV})$ and below $(3666.1 \mathrm{MeV})$ the $\psi^{\prime}$, used for background measurements.

Interesting events are characterized by two nearly back-to-back high energy $e^{ \pm}$. The first level hardware trigger is the logical OR of one main trigger condition and two control triggers 11. The main hardware trigger requires two "electron tracks" as defined by signals in the Cerenkov and corresponding hodoscope elements in coincidence with two back to back energy deposits in the calorimeter. The efficiency of the main trigger was measured on data taken with a dedicated trigger, and continuously monitored by the two control triggers, the first with no Cerenkov requirement and the second with no calorimeter requirement.

As a preliminary selection, all events with $e^{+} e^{-}$candidates with invariant mass $m_{e e}<2.6$ $\mathrm{GeV}$ are rejected.

A maximum likelihood method called "Electron Weight" (EW) has been developed for the rejection of backgrounds that mimic $e^{ \pm}$tracks in the detector (mainly $\gamma$ conversion and $\pi^{0}$ Dalitz decay), comparing the signal and background probability for the candidate $e^{+} e^{-}$pair on the basis of pulse heights in hodoscopes and Cerenkov counters and the CCAL shower shape. Figure 1 shows the logarithm of the EW product for the two $e^{ \pm}$ candidates for data in the $\psi^{\prime}$ energy region and for the background. We choose to cut at $E W_{1} \cdot E W_{2}>1.5$. In this way a clean sample of $e^{+} e^{-}$due to charmonium decay is selected. Figure 2] shows the invariant mass distributions of the $e^{+} e^{-}$candidates before and after the EW cut. After the cut, the two $m_{e e}$ peaks due to the direct decay $\psi^{\prime} \rightarrow e^{+} e^{-}$and the cascade $\psi^{\prime} \rightarrow J / \psi X \rightarrow e^{+} e^{-} X$ are clearly visible.

All the events containing an additional shower in the CCAL within 100 mrad of the $e^{ \pm}$tracks are rejected. This cut yields homogeneous values of the efficiency $\epsilon_{E W}$ for all the final states and reduces the systematic error in the final branching ratio measurements.

$\psi^{\prime} \rightarrow e^{+} e^{-}$and $\psi^{\prime} \rightarrow J / \psi X$ are first selected using kinematical fits requiring $\operatorname{prob}\left(\chi^{2}\right)>10^{-4}$; the $\psi^{\prime} \rightarrow$ $J / \psi X$ are then tagged as $J / \psi \pi^{+} \pi^{-}, J / \psi \pi^{0} \pi^{0}$ or $J / \psi \eta$ with kinematical fit selections using the information on the additional tracks in the detector.

For $J / \psi \pi^{+} \pi^{-}$the $\pi^{ \pm}$tracks are reconstructed within the charged tracking system acceptance $\left(15^{\circ} \leq \theta_{\pi^{ \pm}} \leq\right.$ $55^{\circ}$ ) combining the $\phi$ measurements obtained with the straw chambers ( $\phi$-lines) and the $\theta$ measurements obtained with the scintillating fiber detectors ( $\theta$-lines). For each event containing at least two $\phi$ - and two $\theta$-lines in addition to $e^{+} e^{-}$tracks, all the possible pairs of charged $\theta-\phi$ lines combinations are tested with the kinematical fit as $\pi^{+} \pi^{-}$candidates. The combination with the highest $\chi^{2}$ probability is chosen. The $\pi^{ \pm}$energy cannot be measured in the E835 detector so a $3 \mathrm{C}$ kinematical fit is applied for the selection of $J / \psi \pi^{+} \pi^{-}$events.

The $\pi^{ \pm}$have relatively low momentum in the labo- 


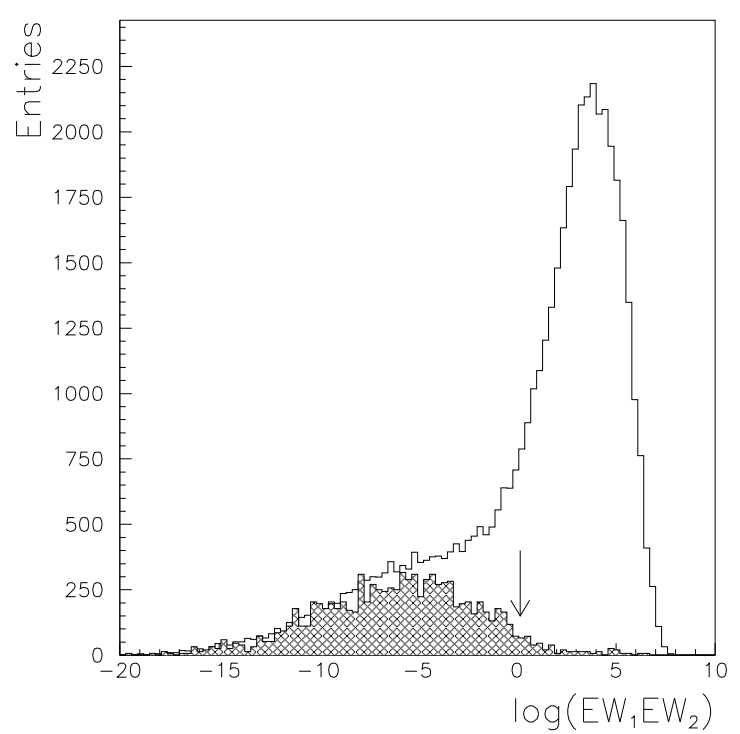

FIG. 1: $\log _{10}\left(E W_{1} \cdot E W_{2}\right)$ distribution for events with $m_{e e}>$ $2.6 \mathrm{GeV}$ in the $\psi^{\prime}$ energy region (open) and on the background with normalized luminosity (cross hatched). The cut $\log _{10}\left(E W_{1} \cdot E W_{2}\right)>\log _{10}(1.5)$ is indicated by the arrow.

ratory and the multiple scattering effect dominates the uncertainty in the measurement of their directions. The associated error has been determined using a full detector simulation based on the GEANT [12] package.

Photons are detected as energy deposits in the CCAL fiducial region $12^{\circ} \leq \theta_{\gamma} \leq 68^{\circ}$. $\pi^{0}$ and $\eta$ candidates are sought in their $\gamma \gamma$ decay, looking for photon pairs of invariant mass between $50 \mathrm{MeV}$ and $350 \mathrm{MeV}$ or 300 $\mathrm{MeV}$ and $800 \mathrm{MeV}$ respectively.

The previous final state categories are mutually exclusive. If an event passes more than one of the previous selections, the event is assigned to the final state with the highest $\operatorname{prob}\left(\chi^{2}\right)$. The number of events selected is reported in Table [. We find that of the 31193 candidate events, $30492(98 \%)$ are selected as $e^{+} e^{-}$or $J / \psi X$, in good agreement with the Monte Carlo results for the efficiencies of the two selections, as described in Section VI]

\section{BACKGROUND SUBTRACTION}

Two sources of background must be subtracted from the observed numbers of events: a non resonant background contribution and an internal background due to final state misidentification.

The non resonant (external) background is due to events not coming from $\psi^{\prime}$ decay. It is measured on data taken in the energy regions far from the resonance (stacks 54a and 54c shown in Table (I) by applying the same analysis as for the $\psi^{\prime}$ peak data; its contribution is com-

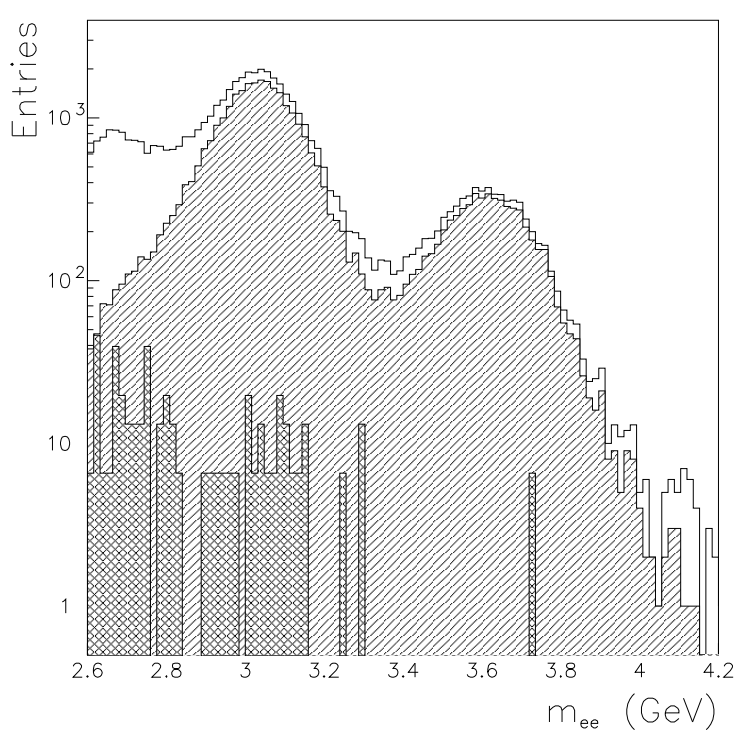

FIG. 2: $m_{e e}$ distribution for all the events with candidate $e^{+} e^{-}$pair with $m_{e e}>2.6 \mathrm{GeV}$ (open). The events selected with the $E W_{1} \cdot E W_{2}>1.5$ cut in the $\psi^{\prime}$ energy region (hatched) and on the background scaled with the luminosity (cross hatched), are also shown.

pared to the resonance signal in Figure 2 The number of background events from this source to be subtracted is obtained scaling the number of events observed in each sample with the integrated luminosity.

The misidentification background is due to resonant events which are not correctly classified. It is evaluated using a Monte Carlo simulation: a sample of events with full detector simulation is generated for all the contaminating channels and analyzed with the same analysis used for the data. The probability of event misidentification is shown in Table III The number of internal background events to be subtracted for each exclusive contaminating channel $\left(N_{\text {int }}(A)\right.$, where $A$ indicates the contaminating final state) is obtained from the number of observed $J / \psi X$ events, scaled with the branching ratio decay mode obtained from the PDG 13], and multiplied by the misidentification probability $P_{\text {misid }}$ (Table 【):

$$
N_{\text {int }}(A)=N(J / \psi X) \cdot\left[\frac{\mathcal{B}\left(\psi^{\prime} \rightarrow A\right)}{\mathcal{B}\left(\psi^{\prime} \rightarrow J / \psi X\right)}\right]_{P D G} \cdot P_{\text {misid }}
$$

The numbers of events after background subtraction are summarized in Table

\section{SELECTION EFFICIENCY}

The overall detection efficiency $\epsilon$ for each exclusive channel is the product of the efficiencies of the cuts used 
TABLE I: Number of events observed in each decay channel in E835 year $2000 \psi^{\prime}$ data, divided by stack. Stacks 54a and 54c are collected far from the resonance and are used for the background measurements. The charged tracking system was off for part of the stack 54a. During this period the $J / \psi \pi^{+} \pi^{-}$could not be detected so the effective total integrated luminosity on the background (stacks $54 \mathrm{a}$ and $54 \mathrm{c}$ ) for this channel is $1.7 \mathrm{pb}^{-1}$.

\begin{tabular}{ccccccc}
\hline \hline Stack & $\mathcal{L}\left(\mathrm{pb}^{-1}\right)$ & Candidates & $e^{+} e^{-}$ & $J / \psi X$ & $J / \psi \pi^{+} \pi^{-}$ & $J / \psi \pi^{0} \pi^{0}$ \\
\hline 1 & 0.748 & 831 & 155 & 658 & 114 & 31 \\
2 & 1.008 & 3595 & 636 & 2893 & 511 & 187 \\
14 & 0.992 & 2248 & 388 & 1807 & 323 & 132 \\
29 & 0.992 & 2083 & 313 & 1706 & 273 & 126 \\
30 & 0.396 & 951 & 158 & 769 & 135 & 45 \\
49 & 2.566 & 5890 & 931 & 4840 & 781 & 322 \\
50 & 1.275 & 3633 & 597 & 2966 & 385 & 205 \\
51 & 2.103 & 5016 & 812 & 4080 & 660 & 275 \\
$54 \mathrm{~b}$ & 2.401 & 6946 & 1114 & 5669 & 971 & 373 \\
\hline $54 \mathrm{a}$ & 1.153 & 35 & 1 & 29 & 1 & 67 \\
$54 \mathrm{c}$ & 0.780 & 23 & 0 & 19 & 1 & 1 \\
\hline \hline
\end{tabular}

TABLE II: Misidentification probabilities. Contaminations smaller than $10^{-3}$ have been neglected.

\begin{tabular}{|c|c|c|c|c|c|}
\hline Generated & $e^{+} e^{-}$ & $J / \psi X$ & $\begin{array}{l}\text { Tagged as } \\
J / \psi \pi^{+} \pi^{-}\end{array}$ & $J / \psi \pi^{0} \pi^{0}$ & $J / \psi \eta$ \\
\hline$e^{+} e^{-}$ & - & $0.038 \pm 0.001$ & $<10^{-3}$ & $<10^{-3}$ & $<10^{-3}$ \\
\hline$J / \psi \pi^{+} \pi^{-} \rightarrow e^{+} e^{-} \pi^{+} \pi^{-}$ & $<10^{-3}$ & - & - & $<10^{-3}$ & $<10^{-3}$ \\
\hline$J / \psi \pi^{0} \pi^{0} \rightarrow e^{+} e^{-} \gamma \gamma \gamma \gamma$ & $<10^{-3}$ & - & $0.0015 \pm 0.0003$ & - & $0.0056 \pm 0.0005$ \\
\hline$J / \psi \eta \rightarrow e^{+} e^{-} \gamma \gamma$ & $<10^{-3}$ & - & $<10^{-3}$ & $<10^{-3}$ & - \\
\hline$J / \psi \eta \rightarrow e^{+} e^{-} \pi^{+} \pi^{-} \pi^{0}$ & $<10^{-3}$ & - & $0.104 \pm 0.002$ & $<10^{-3}$ & $<10^{-3}$ \\
\hline$J / \psi \eta \rightarrow e^{+} e^{-} \pi^{0} \pi^{0} \pi^{0}$ & $<10^{-3}$ & - & $0.0031 \pm 0.0004$ & $0.061 \pm 0.002$ & $<10^{-3}$ \\
\hline$\chi_{c 1} \gamma \rightarrow J / \psi \gamma \gamma \rightarrow e^{+} e^{-} \gamma \gamma$ & $<10^{-3}$ & - & $<10^{-3}$ & $<10^{-3}$ & $0.0150 \pm 0.0008$ \\
\hline$\chi_{c 2} \gamma \rightarrow J / \psi \gamma \gamma \rightarrow e^{+} e^{-} \gamma \gamma$ & $<10^{-3}$ & - & $<10^{-3}$ & $<10^{-3}$ & $<10^{-3}$ \\
\hline
\end{tabular}

for the event selection:

$$
\epsilon=\alpha \cdot \epsilon_{\text {trig }} \cdot \epsilon_{m e e} \cdot \epsilon_{E W} \cdot \epsilon_{\text {sel }},
$$

where $\alpha$ is the acceptance for the $e^{+} e^{-}$pair coming from charmonium decay, $\epsilon_{\text {trig }}$ the trigger efficiency, $\epsilon_{m e e}$ the efficiency of the $e^{+} e^{-}$invariant mass cut $\left(M_{e e}>2.6 \mathrm{GeV}\right)$, $\epsilon_{E W}$ the efficiency of the EW cut $\left(E W_{1} \cdot E W_{2}>1.5\right)$ and $\epsilon_{\text {sel }}$ the final selection efficiency, which includes also the acceptance for all the remaining particles of the final state. The relevant quantity in this analysis is the ratio of the efficiencies for different channels.

\section{A. Acceptance and trigger efficiency}

The final state is characterized by a high invariant mass $e^{+} e^{-}$pair. The hardware $e^{+} e^{-}$trigger is based on the Cerenkov counter, multiplicity and topology in the hodoscopes and multiplicity and topology and energy release in the CCAL.

The $e^{+} e^{-}$acceptance is determined by the Cerenkov fiducial volume, which is defined as $15^{\circ} \leq \theta \leq 60^{\circ}$.

In this analysis, the product $\alpha \cdot \epsilon_{\text {trig }}$ is evaluated for each channel using a sample of Monte Carlo events.

The main source of error on $\alpha \cdot \epsilon_{\text {trig }}$ is the uncertainty in the $e^{+} e^{-}$angular distribution. In the reaction $\bar{p} p \rightarrow$ $\psi^{\prime} \rightarrow e^{+} e^{-}$the $e^{+} e^{-}$pair is distributed according to $1+$ $\lambda_{\psi^{\prime}} \cos \theta_{e}^{*}$, where $\theta_{e}^{*}$ is the angle between the $e^{ \pm}$and the beam directions in the center of mass system and $\lambda_{\psi^{\prime}}$ is the angular distribution parameter. In what follows we used $\lambda_{\psi^{\prime}}=0.67 \pm 0.16$, as recently measured by this experiment [14].

For the decay $\psi^{\prime} \rightarrow J / \psi \pi \pi$, the transition is dominated by the emission of an $L=0$ dipion [15]. In this case the $J / \psi$ has the same polarization as the $\psi^{\prime}$ and the angular distribution for the $J / \psi$ decay is $1+\lambda_{\psi^{\prime}} \cos \theta_{e}^{*}$; in the Monte Carlo simulated events we also assumed $\mathrm{S}$ wave between the dipion and the $J / \psi$.

For the $J / \psi \eta$ channel, the $e^{+} e^{-}$angular distribution is $1+\frac{5}{4} \lambda_{\psi^{\prime}}-\lambda_{\psi^{\prime}} \cos \theta_{e}^{*}$ as discussed in [16].

For the double radiative decay channels $\psi^{\prime} \rightarrow \chi_{c J} \gamma \rightarrow$ $J / \psi \gamma \gamma$, representing a background for some of the channels analyzed here, the $\psi^{\prime}$ radiative decay angular distributions are simulated using the quadrupole amplitudes measured by CBAL [17], while for the $\chi_{c J}$ radiative decays, the angular distributions used in the simulation are the ones measured by E835 [18].

The results obtained for $\alpha \cdot \epsilon_{\text {trig }}$ are summarized in Table IV Using these values we obtained the ratios of 
TABLE III: Numbers of events with statistical errors and background subtraction. $N_{\text {ext }}$ and $N_{\text {int }}$ are the external and internal background contributions.

\begin{tabular}{cccccc}
\hline \hline & $e^{+} e^{-}$ & $J / \psi X$ & $J / \psi \pi^{+} \pi^{-}$ & $J / \psi \pi^{0} \pi^{0}$ & $J / \psi \eta$ \\
\hline$N_{\text {evts }}$ & $5104 \pm 71$ & $25388 \pm 159$ & $4153 \pm 64$ & $1696 \pm 41$ & $363 \pm 19$ \\
$N_{\text {ext }}$ & $7_{-5}^{+15}$ & $316 \pm 46$ & $15_{-10}^{+19}$ & $13_{-9}^{+17}$ & $<11(68 \%$ C.L. $)$ \\
$N_{\text {int }}$ & - & $206 \pm 9$ & $48 \pm 4$ & $28 \pm 2$ & $65 \pm 7$ \\
$N$ & $5097 \pm 73$ & $24866 \pm 166$ & $4090 \pm 67$ & $1655 \pm 44$ & $298 \pm 20$ \\
\hline \hline
\end{tabular}

TABLE IV: $\alpha \cdot \epsilon_{\text {trig }}$ for different values of $\lambda_{\psi^{\prime}}$ obtained with Monte Carlo simulation. The $\chi_{c J} \gamma$ channel, by radiative decay, leads to $J / \psi \gamma \gamma$. The values for the $J / \psi$ inclusive decay are obtained as the weighted average of the exclusive channels.

\begin{tabular}{cccc}
\hline \hline Channel & $\lambda_{\psi^{\prime}}=0.51$ & $\lambda_{\psi^{\prime}}=0.67$ & $\lambda_{\psi^{\prime}}=0.84$ \\
\hline$e^{+} e^{-}$ & 0.5114 & 0.4995 & 0.4893 \\
$J / \psi \pi^{+} \pi^{-}$ & 0.4820 & 0.4681 & 0.4577 \\
$J / \psi \pi^{0} \pi^{0}$ & 0.4831 & 0.4738 & 0.4619 \\
$J / \psi \eta$ & 0.5672 & 0.5751 & 0.5823 \\
$\chi_{c 0} \gamma$ & 0.5249 & 0.5248 & 0.5233 \\
$\chi_{c 1} \gamma$ & 0.5094 & 0.5068 & 0.5030 \\
$\chi_{c 2} \gamma$ & 0.5026 & 0.4937 & 0.4923 \\
$J / \psi X$ & 0.4890 & 0.4785 & 0.4692 \\
\hline \hline
\end{tabular}

the $\alpha \cdot \epsilon_{\text {trig }}$ product between different channels, namely:

$$
\begin{aligned}
\frac{\alpha \cdot \epsilon_{\text {trig }}(J / \psi X)}{\alpha \cdot \epsilon_{\text {trig }}\left(e^{+} e^{-}\right)} & =0.9579 \pm 0.0016, \\
\frac{\alpha \cdot \epsilon_{\text {trig }}(J / \psi X)}{\alpha \cdot \epsilon_{\text {trig }}\left(J / \psi \pi^{+} \pi^{-}\right)} & =1.022 \pm 0.007, \\
\frac{\alpha \cdot \epsilon_{\text {trig }}(J / \psi X)}{\alpha \cdot \epsilon_{\text {trig }}\left(J / \psi \pi^{0} \pi^{0}\right)} & =1.010 \pm 0.006, \\
\frac{\alpha \cdot \epsilon_{\text {trig }}(J / \psi X)}{\alpha \cdot \epsilon_{\text {trig }}(J / \psi \eta)} & =0.83 \pm 0.03,
\end{aligned}
$$

where the error is systematic and comes from the uncertainty in the $e^{+} e^{-}$angular distributions.

\section{B. $e^{+} e^{-}$invariant mass cut efficiency}

The efficiency of the $e^{+} e^{-}$invariant mass cut, $m_{e e}>$ $2.6 \mathrm{GeV}$, is $\epsilon_{\text {mee }} \simeq 96 \%$, and affects all the channels in the same way, except $\psi^{\prime} \rightarrow e^{+} e^{-}$which has a higher efficiency because of the higher $e^{+} e^{-}$invariant mass. The efficiency ratio is determined from Monte Carlo to be $\frac{\epsilon_{m e e}\left(\psi^{\prime} \rightarrow J / \psi X \rightarrow e^{+} e^{-} X\right)}{\epsilon_{m e e}\left(\psi^{\prime} \rightarrow e^{+} e^{-}\right)}=0.992 \pm 0.001$.

\section{Electron weight cut efficiency}

The presence of additional showers in the CCAL in proximity to the $e^{ \pm}$tracks modifies the CCAL shower shape for the $e^{ \pm}$, resulting in a lower EW cut efficiency for these events. The events containing extra CCAL showers within $100 \mathrm{mrad}$ of $e^{ \pm}$tracks are rejected; this allows the use of the same EW efficiency for all channels. The residual fluctuations of the $\epsilon_{E W}$ values for different channels, due to additional effects, have been studied with the Monte Carlo and are within 2\%, which has been taken as the EW cut systematic error.

\section{Final selection efficiency}

The effect of the $100 \mathrm{mrad}$ cut in the CCAL, the acceptance for exclusive reactions, and the kinematic fit selection efficiency for each analyzed channel are included in the final selection efficiency $\epsilon_{\text {sel }}$. This is determined from Monte Carlo event samples by performing the same cuts applied on data. The systematic error associated with the efficiency is obtained by trying various $\operatorname{prob}\left(\chi^{2}\right)$ cuts in the range $10^{-4}-10^{-1}$ for the $J / \psi \eta$ channel and $10^{-6}$ $10^{-2}$ for all the other channels, for both data and Monte Carlo events, and observing the result fluctuations. The results obtained for each channel are shown in Table $\nabla$

\section{RESULTS}

Using the efficiency values obtained in the previous section and (2) we obtain:

$$
\begin{array}{r}
\mathcal{B}\left(\psi^{\prime} \rightarrow e^{+} e^{-}\right) \\
\mathcal{B}\left(\psi^{\prime} \rightarrow J / \psi X\right) \cdot \mathcal{B}\left(J / \psi \rightarrow e^{+} e^{-}\right) \\
=0.206 \pm 0.003 \pm 0.008, \\
\frac{\mathcal{B}\left(\psi^{\prime} \rightarrow J / \psi \pi^{+} \pi^{-}\right)}{\mathcal{B}\left(\psi^{\prime} \rightarrow J / \psi X\right)} \\
=0.525 \pm 0.009 \pm 0.022, \\
\mathcal{B}\left(\psi^{\prime} \rightarrow J / \psi \pi^{0} \pi^{0}\right) \cdot \mathcal{B}\left(\pi^{0} \rightarrow \gamma \gamma\right)^{2} \\
\hline \mathcal{B}\left(\psi^{\prime} \rightarrow J / \psi X\right) \\
=0.292 \pm 0.008 \pm 0.019, \\
\mathcal{B}\left(\psi^{\prime} \rightarrow J / \psi \eta\right) \cdot \mathcal{B}(\eta \rightarrow \gamma \gamma) \\
\mathcal{B}\left(\psi^{\prime} \rightarrow J / \psi X\right) \\
=0.0197 \pm 0.0013 \pm 0.0013,
\end{array}
$$

where the first error is statistical and the second systematic.

The number of events observed in the $J / \psi \pi^{0} \pi^{0}$ and $J / \psi \eta$ decay modes could also be normalized to the events 
TABLE V: $\epsilon_{\text {sel }}$ for each decay channel. The error is systematic and is obtained by examining $\operatorname{several~prob}\left(\chi^{2}\right)$ cuts.

\begin{tabular}{cccccc}
\hline \hline Channel & $e^{+} e^{-}$ & $J / \psi X$ & $J / \psi \pi^{+} \pi^{-}$ & $J / \psi \pi^{0} \pi^{0}$ & $J / \psi \eta$ \\
\hline$\epsilon_{\text {sel }}$ & $0.943 \pm 0.031$ & $0.996 \pm 0.011$ & $0.319 \pm 0.011$ & $0.229 \pm 0.014$ & $0.502 \pm 0.024$ \\
\hline \hline
\end{tabular}

observed in the $J / \psi \pi^{+} \pi^{-}$. Our result for these decay modes could alternatively be expressed as:

$$
\frac{\mathcal{B}\left(\psi^{\prime} \rightarrow J / \psi \pi^{0} \pi^{0}\right)}{\mathcal{B}\left(\psi^{\prime} \rightarrow J / \psi \pi^{+} \pi^{-}\right)}=0.571 \pm 0.018 \pm 0.044
$$

to be compared with the value 0.5 expected from isospin conservation, and:

$$
\frac{\mathcal{B}\left(\psi^{\prime} \rightarrow J / \psi \eta\right)}{\mathcal{B}\left(\psi^{\prime} \rightarrow J / \psi \pi^{+} \pi^{-}\right)}=0.095 \pm 0.007 \pm 0.007
$$

in agreement with theoretical estimates [5, [6].

From our measurement and the world averages for $\mathcal{B}\left(\psi^{\prime} \rightarrow J / \psi X\right)=0.557 \pm 0.026, \mathcal{B}\left(J / \psi \rightarrow e^{+} e^{-}\right)=$ $0.0593 \pm 0.0010, \mathcal{B}\left(\pi^{0} \rightarrow \gamma \gamma\right)=0.98798 \pm 0.00032$ and $\mathcal{B}(\eta \rightarrow \gamma \gamma)=0.3943 \pm 0.0026$ [13] we can derive the following values for the $\psi^{\prime}$ branching ratios:

$$
\begin{aligned}
\mathcal{B}\left(\psi^{\prime} \rightarrow e^{+} e^{-}\right) & =(6.8 \pm 0.1 \pm 0.4) \times 10^{-3} \\
\mathcal{B}\left(\psi^{\prime} \rightarrow J / \psi \pi^{+} \pi^{-}\right) & =0.292 \pm 0.005 \pm 0.018 \\
\mathcal{B}\left(\psi^{\prime} \rightarrow J / \psi \pi^{0} \pi^{0}\right) & =0.167 \pm 0.005 \pm 0.014 \\
\mathcal{B}\left(\psi^{\prime} \rightarrow J / \psi \eta\right) & =0.028 \pm 0.002 \pm 0.002
\end{aligned}
$$

The results are in excellent agreement with the recent measurements published by BES 19] 20]. The present result is compared with the ones obtained previously by our experiment and E760 in Table VI The larger systematic error in the present results comes from a more conservative evaluation of the kinematic fit systematic.

\section{DIPION INVARIANT MASS DISTRIBUTION MEASUREMENT}

The dipion invariant mass $\left(m_{\pi \pi}\right)$ distributions for $J / \psi \pi^{+} \pi^{-}$and $J / \psi \pi^{0} \pi^{0}$ events, corrected for the detector acceptance, are shown in Figure 3. 7]:

A possible parametrization for the $m_{\pi \pi}$ distribution is

$$
\frac{d \Gamma}{d m_{\pi \pi}} \propto P_{S} \cdot\left(m_{\pi \pi}^{2}-\Lambda_{\pi \pi} m_{\pi}^{2}\right)^{2}
$$

where the phase space $P_{S}$ is:

$$
P_{S}=\sqrt{\frac{\left(m_{\pi \pi}^{2}-4 m_{\pi}^{2}\right)\left[M_{J / \psi}^{4}+M_{\psi^{\prime}}^{4}+m_{\pi \pi}^{4}-2\left(M_{J / \psi}^{2} m_{\pi \pi}^{2}+M_{\psi^{\prime}}^{2} m_{\pi \pi}^{2}+M_{J / \psi}^{2} M_{\psi^{\prime}}^{2}\right)\right]}{4 M_{\psi^{\prime}}^{2}}} .
$$

The measured $m_{\pi^{+} \pi^{-}}$and $m_{\pi^{0} \pi^{0}}$ distributions, corrected for the detector acceptance, are fitted to the function (19) convoluted with their resolution as determined by Monte Carlo.

The fitting procedure has been verified to reproduce the correct (input) result when applied to simulated events, and to perform equally well on the neutral and charged dipion modes.

The results obtained for the $m_{\pi \pi}$ distribution are $\Lambda_{\pi^{+} \pi^{-}}=3.31 \pm 0.15_{-0.15}^{+0.35}\left(\chi^{2} / n d f=32.3 / 29=1.1\right)$ and $\Lambda_{\pi^{0} \pi^{0}}=4.06 \pm 0.25_{-0.15}^{+0.25}\left(\chi^{2} / n d f=54.4 / 29=1.9\right)$ where the first error is statistical and the second systematic. The $m_{\pi \pi}$ distributions for $J / \psi \pi^{+} \pi^{-}$and $J / \psi \pi^{0} \pi^{0}$ are expected to be the same.

For the evaluation of the systematic error, several fits to the data have been done, varying the binning, the parameters of the resolution function and the acceptance correction factors. The value of $\Lambda_{\pi \pi}$ was found to be very sensitive to the parameters of the resolution function, which is described by a double gaussian whose parameters are obtained from the Monte Carlo. The systematic errors have been obtained by varying the parameters of the distribution to take into account possible discrepancies between the data and the Monte Carlo. For the acceptance correction factors, some sets of values have been calculated under the hypothesis of $\Lambda_{\pi \pi}$ values from 2 to 6 . Then the data have been fitted using different corrections; the $\Lambda_{\pi \pi}$ values fitted on data using the different correction factors show variations by a fraction of the statistical error, and so no systematic error is associated with the acceptance correction. We also observe that the systematic uncertainty introduced by $\Lambda_{\pi \pi}$ on the acceptance used in the measurement of the branching ratios of the $J / \psi \pi \pi$ channels is negligible with respect to the ones in Tables IV and V

The only other available measurement of $\Lambda_{\pi \pi}$ has been 
TABLE VI: $\psi^{\prime}$ BR measurements obtained by E835.

\begin{tabular}{cccccc}
\hline \hline & $\frac{\mathcal{B}\left(\psi^{\prime} \rightarrow e^{+} e^{-}\right)}{\mathcal{B}\left(\psi^{\prime} \rightarrow J / \psi X\right)}$ & $\frac{\mathcal{B}\left(\psi^{\prime} \rightarrow J / \psi \pi^{+} \pi^{-}\right)}{\mathcal{B}\left(\psi^{\prime} \rightarrow J / \psi X\right)}$ & & $\frac{\mathcal{B}\left(\psi^{\prime} \rightarrow J / \psi \pi^{0} \pi^{0}\right)}{\mathcal{B}\left(\psi^{\prime} \rightarrow J / \psi X\right)}$ & $\frac{\mathcal{B}\left(\psi^{\prime} \rightarrow J / \psi \eta\right)}{\mathcal{B}\left(\psi^{\prime} \rightarrow J / \psi X\right)}$ \\
\hline E760[1] & $0.0144 \pm 0.0008$ & $0.496 \pm 0.037$ & $0.323 \pm 0.033$ & $0.061 \pm 0.009$ \\
E835[2] & $0.0128 \pm 0.0004$ & - & $0.328 \pm 0.015$ & $0.072 \pm 0.009$ \\
E835 (this paper) & $0.0122 \pm 0.0002 \pm 0.0005$ & $0.525 \pm 0.009 \pm 0.022$ & $0.300 \pm 0.008 \pm 0.022$ & $0.050 \pm 0.006 \pm 0.003$ \\
\hline \hline
\end{tabular}
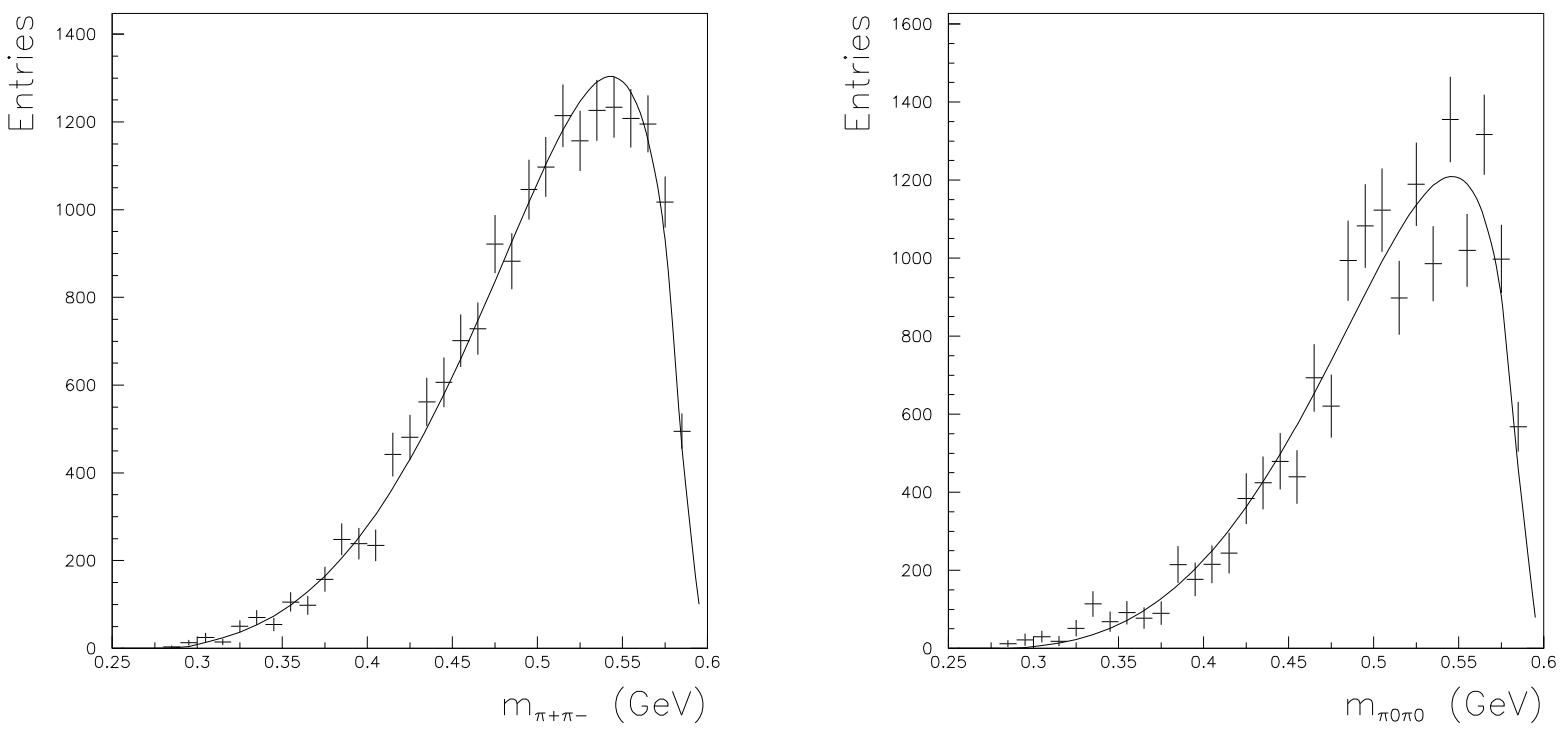

FIG. 3: $\pi \pi$ invariant mass distribution for (a) $J / \psi \pi^{+} \pi^{-}$, (b) $J / \psi \pi^{0} \pi^{0}$ events, corrected for the acceptance. The solid line is the fit with $P_{S} \cdot\left(m_{\pi \pi}^{2}-\Lambda_{\pi \pi} m_{\pi}^{2}\right)^{2}$.

obtained by BES for the decay $\psi^{\prime} \rightarrow J / \psi \pi^{+} \pi^{-}$, which yielded $\Lambda_{\pi^{+} \pi^{-}}=4.35 \pm 0.06 \pm 0.17$ [15]. The fit to our data using this value yields a $\chi^{2} / n d f=2.7$.

\section{Acknowledgments}

The authors wish to thank the staffs, engineers and technicians at their respective institutions for their valu- able help and cooperation. This research was supported by the U.S. Department of Energy and by the Italian Istituto Nazionale di Fisica Nucleare.
[1] T. A. Armstrong et al. [Fermilab E760 Collaboration], Phys. Rev. D 55 (1997) 1153.

[2] M. Ambrogiani et al. [E835 Collaboration], Phys. Rev. D 62 (2000) 032004.

[3] H. Goldberg, Phys. Rev. Lett. 35 (1975) 605.

[4] K. Gottfried, Phys. Rev. Lett. 40 (1978) 598.

[5] M. B. Voloshin and V. I. Zakharov, Phys. Rev. Lett. 45 (1980) 688.

[6] V. A. Novikov and M. A. Shifman, Z. Phys. C 8 (1981) 43.

[7] T. N. Pham, B. Pire and T. N. Truong, Phys. Lett. B 61
(1976) 183.

[8] T. M. Yan, Phys. Rev. D 22 (1980) 1652.

[9] G. Garzoglio et al. [E835 Collaboration], Nucl. Instrum. Meth. A 519 (2004) 558.

[10] D. P. McGinnis, G. Stancari and S. J. Werkema, Nucl. Instrum. Meth. A 506 (2003) 205.

[11] W. Baldini, D. Bettoni, R. Calabrese, E. Luppi, R. Mussa and G. Stancari, Nucl. Instrum. Meth. A 449 (2000) 331.

[12] GEANT - Detector Description and Simulation Tool, http://wwwinfo.cern.ch/asd/geant/

[13] K. Hagiwara et al. [Particle Data Group Collaboration], 
Phys. Rev. D 66 (2002) 010001.

[14] M. Ambrogiani et al. [Fermilab E835 Collaboration], arXiv:hep-ex/0412007.

[15] J. Z. Bai et al. [BES Collaboration], Phys. Rev. D 62 (2000) 032002.

[16] A. J. Smith, Ph. D. thesis, University of California, Irvine (1993).

[17] M. Oreglia et al., Phys. Rev. D 25 (1982) 2259.
[18] M. Ambrogiani et al. [E835 Collaboration], Phys. Rev. D 65 (2002) 052002.

[19] M. Ablikim et al. [BES Collaboration], Phys. Rev. D 70 (2004) 012003.

[20] J. Z. Bai et al. [BES Collaboration], Phys. Rev. D 70 (2004) 012006 